ACTA MYCOLOGICA

Vol. 49 (1): 99-107

2014
Dedicated to Professor Maria Ławrynowicz on the occasion of the 45th anniversary of her scientific activity

DOI: $10.5586 / \mathrm{am} .2014 .012$

\title{
Diversity of macromycetes in the Białaczów nature reserve (Central Poland)
}

\section{AGNIESZKA SALAMAGA ${ }^{1}$ and DOMINIKA ŚLUSARCZYK ${ }^{2}$}

\author{
${ }^{1}$ Jagiellonian University, Institute of Botany \\ Kopernika 27, PL-31-501 Kraków, asalamaga@wp.pl \\ ${ }^{2}$ University of Lodz, Faculty of Biology and Environmental Protection \\ Department of Algology and Mycology, Banacha 12/16, PL-90-237 Łódź, dominika@biol.uni.lodz.pl
}

Salamaga A., Ślusarczyk D.: Diversity of macromycetes in the Białaczów nature reserve (Central Poland). Acta Mycol. 49 (1): 99-107, 2014.

The paper contains preliminary results of mycological research conducted in the Białaczów forest reserve, situated at the Wzgórza Opoczyńskie Upland. There are only data on macromycetes at this area. The occurrence of 167 species of macrofungi has been documented on the background of variety of tree stand and habitats. In July 2011 a heavy wind storm destroyed the tree stand and devastated the reserve. Now the area is in course of natural renovation. The data presented in the paper could be used as comparative fungal material concerning qualitative and quantitative changes.

Key words: macrofungi, mycological research, rare species, protected area

\section{INTRODUCTION}

The topic of the study are macrofungi in Białaczów forest reserve, in reference to tree stands and habitat background. Mycological studies in Central Poland were initiated by Ławrynowicz (1973) and conducted in over 40 natural reserves (Lawrynowicz 2002; Szkodzik 2005; Adamczyk 2007).

The Białaczów reserve is one of those reserves from which collections were not published jet. The fact that it was mostly destroyed during a wind storm in July 2011, led to the presentation of the existing material. The obtained preliminary results, and the gathered herbarium documentation will be the basis for comparison and confrontation of the changes that occurred in the fungal diversity after the storm. 


\section{STUDY AREA}

The Białaczów forest reserve (Łódź Province, Opoczno Forestry District, N 51¹8, E $20^{\circ} 17$ ) covers 21,87 ha and was created on 24.05.1976, it's main goal being the preservation of a wet-ground forest with it's natural characteristics (Rąkowski 2006). The reserve contains a whole forestry unit (101). The borders of the reserve consist of a road leading from Miedzna Drewniana to Białaczów in the south and west, a small watercourse with swampy meadows in the north, and lands belonging to the Social House in Białaczów in the east (Fig. 1).

In according to geographical classification, the reserve is located in Wzgórza Opoczyńskie Upland, surrounding the Świętokrzyskie Mountains from North West.

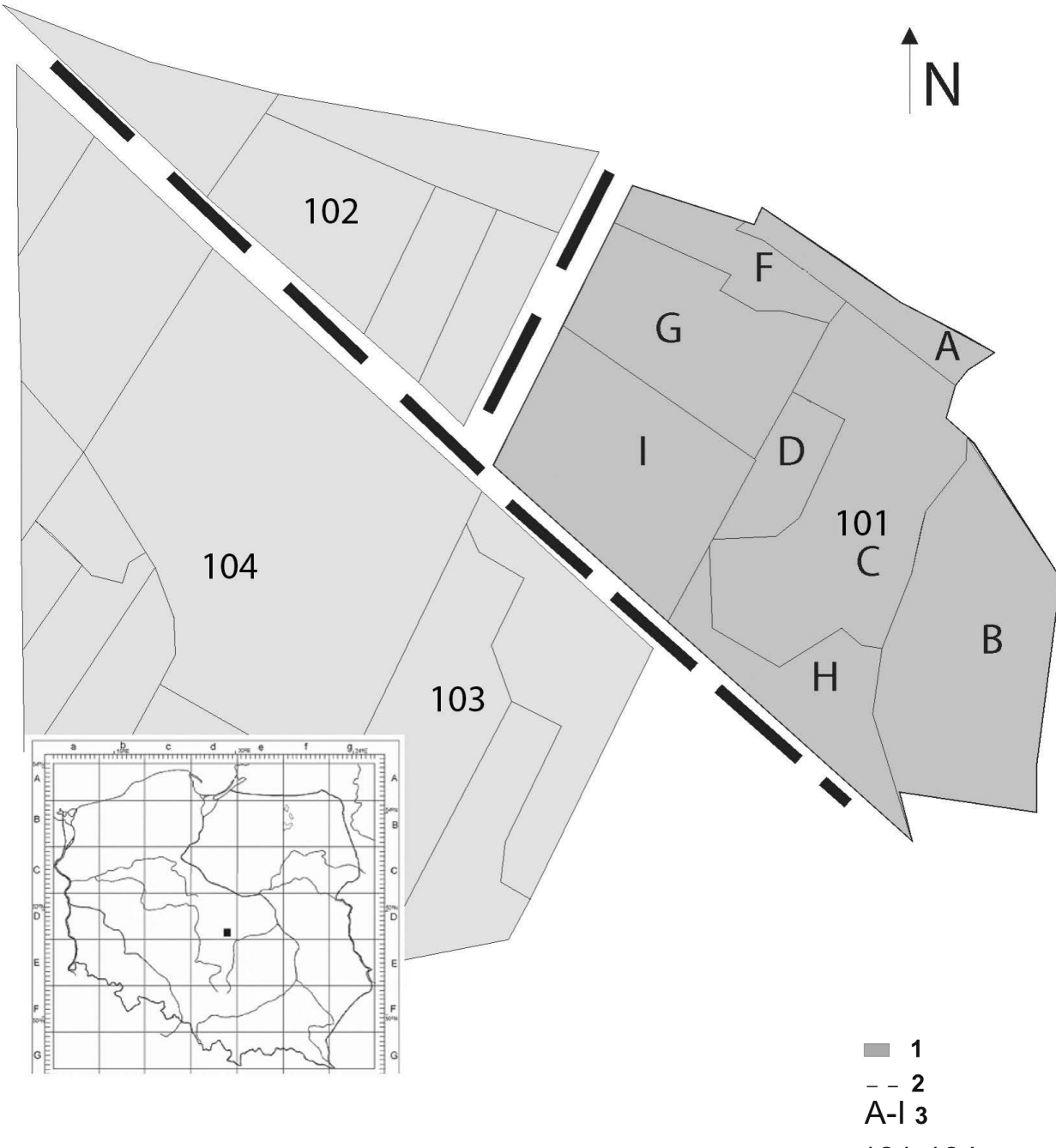

101-104 4

Fig. 1. Study area: 1 - the Białaczów reserve, 2 - roads, 3 - forest sections, 4 - forest units. 
The upland consist of Jurassic rock, on which lay gravel monadnocks (Kondracki 2002). In the reserve two types of soil are distinguished: muck-gleyed soil in the subunit a, and both brown and poor sand-based postglacial soil in the rest of the area (Operat Urządzeniowy 1998).

The dominant plant community in the reserve is Tilio-Carpinetum calamagrostietosum and small fragments of natural riparian forests: Fraxino-Alnetum and FicarioUlmetum. Tree species occurring there are: Alnus glutinosa, Fraxinus excelsior, Ulmus campestris, Acer pseudoplatanus, A. platanoides, Carpinus betulus. In Tilio-Carpinetum calamagrostietosum apart from species Tilia cordata, Fagus sylvatica, Quercus robur and Carpinus betulus, there are also those that were introduced into the treestand: Betula pendula, Pinus sylvestris, Robinia pseudoacacia, Quercus rubra and $Q$. palustris. Hepatica nobilis, a species under strict protection have been found in the reserve, and also species under partial protection: Convallaria majalis, Hedera helix, and Pleurozium schreberi in the moss layer.

\section{MATERIAL AND METHODS}

Mycological studies in the reserve were conducted in years 2006-2007, using the route method, and the permanent study plots.

The following monographs and keys were used in fungi identification: Skirgiełło (1960, 1991, 1998), Domański (1965), Jülich (1984), Galli (1996), Nespiak (1981, 1990), Moser (1978), Breitenbach, Kränzlin (1984, 1986, 1991, 1995, 2000), Knudsen, Vesterholt (2008) and Noordeloos et al. (2001, 2005). Standard methods of studying macrofungi, based on microscopic analyses, were used in the species identification (hand-made sections or squash preparations mounted in water, 3-5\% $\mathrm{KOH}$, Melzer's reagent; light microscope).

The nomenclature follows Wojewoda (2003), and Index Fungorum (2013). Threat categories are listed according to Wojewoda and Ławrynowicz (2006). The specimens are deposited in the Herbarium Universitatis Lodziensis (LOD - F).

\section{RESULTS AND NOTES}

Presented list contains first mycological data from Wzgórza Opoczyńskie Upland. In total, 167 taxa of macomycetes (species and forms) including 13 Ascomycota and 154 Basidiomycota were identified.

Most of the gathered species were saprotrophic fungi - 118 species, which make $70 \%$ of the studied material. This shows an adequate amount of diverse substratum (leaves, needles, cones, small branches) needed for fungal growth in this habitat. 44 of gathered species were mycorrhizal fungi, and 5 were parasites. From ecological point of view, most of the gathered fungi produce fruit bodies above ground (74) and on wood (62), wheares fungi on plant litter were represented by 31 species.

The following abbreviations are used in the list of species: T-C - Tilio-Carpinetum, F-U - Ficario-Ulmetum, F-A - Ficario-Almetum, R - Red List Category. 


\title{
LIST OF SPECIES
}

\author{
ASCOMYCOTA
}

\author{
Ascocoryne sarcoides (Jacq.) J.W. Groves \& D.E. Wilson - on wood, T-C, VIII-XI 2006-2007. \\ Bisporella citrina (Batsch) Korf \& S.E. Carp. - on deciduous wood, T-C, V 2007. \\ Bulgaria inquinans (Pers.) Fr. - on deciduous wood, T-C, IX 2007. \\ Elaphomyces granulatus Fr emend. Holl. - in deciduous and mixed tree stands, in soil (Lawrynowicz 1989). \\ Hypoxylon deustum (Hoffm.) Grev. - on dead wood, T-C, IX 2007. \\ Hypoxylon fragiforme (Pers.) J. Kickx - on dead wood, T-C, III-XII 2006-20087 \\ Lasiosphaeria ovina (Pers.) Ces. \& De Not. - on dead wood, T-C, VII 2007. \\ Mollisia amenticola (Sacc.) Rehm - on wood, T-C, XI 2007. \\ Nectria cinnabarina (Tode) Fr. - on wood, T-C, F-U, F-A, VIII 2007. \\ Otidea onotica (Pers.) Fuckel - on the ground, T-C, VIII 2007. \\ Pachyphloeus melanoxanthus Tul. - under Fagus sylvatica, in soil (Lawrynowicz 1990), R. \\ Scutellinia scutellata (L.) Lambotte - on wood, T-C, VI 2006-2007. \\ Trichophaea hemisphaerioides (Mouton) Graddon - on the ground, T-C, IX 2007. \\ Xylaria hypoxylon (L.) Grev. - on dead wood, T-C, IX-XI 2007,VI-XI 2007. \\ Xylaria polymorpha (Pers.) Grev. - on dead wood, T-C, X-XII 2006-2007.
}

\section{BASIDIOMYCOTA}

Agaricus silvaticus Schaeff. - on the ground, T-C, X 2007.

Agaricus silvicola (Vittad.) Peck. - on the ground, T-C, IX-X 2007.

Amanita citrina (Schaeff.) Pers. - on the ground, T-C, F-U, VIII-X 2007.

Amanita citrina var. alba (Gillet) Gilbert. - on the ground, T-C, X 2007.

Amanita muscaria (L. Fr.) Hook. - on the ground, T-C, IX-X 2007.

Amanita pantherina (DC.) Krombh. - on the ground, T-C, IX-X 2007.

Amanita porphyria (Alb \& Schwein.: Fr) Mladý. - on the ground, T-C, IX 2007.

Armillaria mellea (Vahl.: Fr.) P. Kumm. s.str. - on dead wood, T-C, X 2007.

Auricularia auricula-judae (Bull.: Fr.). Wettst - on a branch of a deciduous tree, T-C, F-U, III-XI 2007.

Auriscalpium vulgare Gray - on pine cone, T-C, IX-XI 2007.

Boletus edulis Bull.: Fr. - on the ground, T-C, IX 2007.

Calocera viscosa (Pers.: Fr.) Fr. - on conifers stump, T-C, VI-VII 2007.

Cantharellus cibarius Fr. - on the ground, T-C, VII-X 2007.

Chroogomphus rutilus (Schaeff.: Fr.) O. K. Miller - on the ground, T-C, X 2007.

Clavariadelphus fistulosus (Holmsk.) Corner - on twigs of deciduous tree, T-C, XI 2007, R.

Clavariadelphus junceus (Alb \& Schwein.: Fr.) Corner - on fallen leaves of Betula pendula, T-C, X 2007, R.

Clitocybe candicans (Pers.) P. Kumm. - on the ground, T-C, X 2007.

Clitocybe clavipes (Pers.: Fr.) P. Kumm. - on the ground, T-C, X 2007.

Clitocybe gibba (Pers.: Fr.) P. Kumm. - on the ground, T-C, X 2007.

Citocybe nebularis (Batsch) P. Kumm. - on the ground, F-U, X-XI 2007.

Clitocybe odora (Bull.: Fr.) P. Kumm. - on the ground, T-C, VIII 2007.

Clitocybe phyllophila ( Fr.) P. Kumm. - on litter, T-C, F-U, X 2007.

Coprinus micaceus (Bull.: Fr.) Fr. - around stumps and logs of deciduous tree, T-C, F-U, X 2007.

Coprinus truncorum (Scop.) Fr. ss. Romagn. - on stumps and logs on dead wood, F-U, VII 2007.

Cortinarius alboviolaceus (Pers.: Fr.) Fr. - on the ground, T-C, X 2007.

Cortinarius hemitrichus (Pers.: Fr.) Fr. - on the ground, T-C, X 2007.

Crepidotus variabilis (Pers.: Fr.) P. Kumm. - on dead fallen twigs of deciduous tree, T-C, F-U, IX-X 2007.

Crucibulum laeve (Huds.) Kambly. - on a branch in the litter, T-C, IX 2007.

Cyathus striatus (Huds.)Willd. - on a branch in the litter, T-C, F-U, X 2007.

Cystoderma amianthinum (Scop.: Fr.) Fayod - of mosses, T-C, X 2007. 
Dacrymyces stillatus Nees: Fr. - on the branch, T-C, F-U, V-XII 2007.

Daedaleopsis confragosa (Bolt.: Fr.) J. Schröt. - on a branch of a deciduous tree, T-C, IX-XI 2007. Exidia glandulosa (Bull.): Fr. - on a branch of a deciduous tree, T-C, F-U, III-XI 2007.

Exidia plana (Wiggers) Donk. - on dead wood, T-C, X 2007.

Exidia saccharina (Alb. \& Schwein.) Fr. - on wood of Pinus sylvestris, T-C XI 2007.

Flammulina velutipes (M.A. Curtis: Fr) Singer - on deciduous tree stump, T-C, XI-XII 2007.

Fomes fomentarius (L.: Fr.) Kickx. - on Betula pendula, T-C, F-U, V-XII 2007.

Fomitopsis pinicola (Swart: Fr..) P. Karst. - on coniferous tree stump, T-C, V-XII 2007.

Galerina pumila (Pers.: Fr.) Singer - among grass andt mosses, T-C, X 2007.

Ganoderma applanatum (Per.) Pat. - on deciduous tree stump, T-C, XI-XII 2007.

Gymnopus confluens (Pers.) P. Kumm. - on litter, T-C, F-U, VI-VIII 2007.

Gymnopus dryophilus (Bull.) P. Kumm. - on litter, T-C, X 2007.

Gymnopus peronatus (Bolt. Fr.) Antonin, Halling \& Noordel. - on litter, T-C, F-U, VII-X 2007.

Gyroporus cyanescens (Bull.: Fr.) Quél. - on the ground, T-C, VII 2007, R.

Hapalopilus rutilans (Pers.) P. Karst. - on trunks and branches of deciduous tree, T-C, VI 2007.

Hebeloma mesophaeum (Pers.) Quél. - on the ground, T-C, X 2007.

Heterobasidion annosum (Fr.) Bref. sl. - on Pinus sylvestris stump, T-C, XI 2007.

Hochenbuehelia grisea (Peck) Singer - on a branch of a deciduous tree, T-C, X 2007.

Hygrophoropsis aurantiaca (Wulf.: Fr.) J. Schröt. - on the ground, T-C, IX-X 2007.

Hygrophorus hypothejus var. hypothejus (Fr.: Fr.) Fr. - on the ground in sandy soil, T-C, XI 2007.

Hymenochaete rubiginosa (Schrad.: Fr.) Lév. - on deciduous tree stump, T-C, VI 2007.

Hypsizygus ulmarius (Bull.: Fr.) Redhead - on living trunk of Ulmus laevis F-U, X-XI 2007, V.

Inonotus tomentosus (Fr.) Teng - on the roots of conifers, T-C, VII 2007, V.

Laccaria amethystina Cooke - on the ground, T-C, IX-X 2007.

Laccaria bicolor (Maire) P.D. Orton - on the ground, T-C, IX-X 2007.

Laccaria laccata (Scop.: Fr.) Berk. \& Broome - on the ground, T-C, X 2007.

Lactarius camphoratus Fr. - on the ground, T-C, VIII 2007.

Lactarius chrysorrheus Fr. - on the ground, T-C, X 2007, R.

Lactarius quietus (Fr.) Fr. - on the ground, T-C, X 2007.

Lactarius thejogalus (Bull.: Fr.) Gray ss. Neuhoff. - on the ground, T-C, X 2007.

Lactarius torminosus (Schaeft.: Fr.) Pers. - on the ground, T-C, IX 2007.

Lactarius vellereus (Fr.) Fr. - on the ground, T-C, VIII 2007.

Laetiporus sulphureus (Bull.: Fr.) Murrill - on deciduous tree, T-C, VIII 2007.

Leccinum scabrum (Bull.: Fr.) Gray - on the ground, T-C, IX-X 2007.

Lentinellus cochleatus (Pers.: Fr.) P. Karst. - on deciduous tree stump, T-C, F-U, IX-X 2007.

Lepiota cristata J.E. Lange - on the ground, T-C, IX 2007.

Lepiota perplexa Knudsen - on the ground, T-C, VII 2007.

Lepista flaccida (Sowerby: Fr.) Pat. - on the ground, T-C, X-XII 2007.

Lepista gilva (Pers.: Fr.) Pat. - on the ground, T-C, X 2007.

Lepista nuda (Bull.: Fr.) Cooke. - on the ground, T-C, F-U, IX-X 2007.

Lycoperdon molle Pers. - on the ground, T-C, VIII 2007.

Lycoperdon nigrescens (Pers.: Pers.) Pers. - on the ground, T-C, F-U, IX-X 2007.

Lycoperdon perlatum (Pers.: Pers). - on the ground, T-C, F-U, VIII-X 2007.

Macrolepiota procera (Scop.: Fr.) Singer - on the ground, T-C, IX 2007.

Macrolepiota rhacodes (Vittad.) Singer - on the ground, T-C, IX-X 2007.

Marasmius alliaceus (Jacq.: Fr.) Fr. - on the ground, T-C, F-U, IX-X 2007.

Marasmius rotula (Scop.: Fr.) Fr - on twigs of Carpinus betulus, T-C, VII 2007.

Marasmius scorodonius (Fr.: Fr.) Fr. - on fallen twigs of Pinus, T-C, X-XII 2007.

Melanoleuca schumacheri (Fr.: Fr.) Singer - on the ground, T-C, X 2007.

Note. Species recorded in Poland from few sites (Wojewoda 2003; Flisińska 2004; Bujakiewicz 2010).

Mycena arcangeliana Bres. - on decayedtrunks and stumps on deciduous trees, T-C, XI 2007.

Mycena cinerella (P. Karst.) P. Karst. - among mosses, T-C, XI 2007.

Mycena epipterygia ( Scop. Fr.) Gray - on plant debris and fragments of grass, T-C, X 2007.

Mycena galericulata (Scop.: Fr.) - dead trunks, T-C, VIII 2007.

Mycena galopus (Pers.: Fr.) P. Kumm. - among mosses, T-C, VII 2007.

Mycena inclinata (Fr.) Quél. - on stumps and trunks of deciduous trees, T-C, F-U, VII-XI 2007. 
Mycena leptocephala (Pers.: Fr.) Millet - in liter, T-C, VII-X 2007.

Mycena maculata P. Karst. - on rotten stumps, T-C, F-U, X-XI 2007.

Mycena polygramma (Bull.: Fr.) Gray - on stumps of deciduous trees, T-C, X 2007.

Mycena pura (Pers. Fr.) P. Kumm. - in litter, T-C, F-U, IX-X 2007.

Mycena purpurefusca (Peck) Sacc. - in litter, T-C, X 2007.

Mycena sanguinolenta (Alb. \& Schwein.: Fr.) P. Kumm. - on litter, T-C, X-XI 2007.

Mycena stylobates (Pers.: Fr.) P. Kumm. - of fallen leaves of Quercus robur, T-C, F-U 2007.

Mycena viridimarginata P. Karst. - in litter, T-C, X 2007.

Mycena vitilis (Fr.) Quél. - in litter, T-C, F-U, IX-X 2007.

Mycena vulgaris (Pers.: Fr. ) P. Kumm. - among mosses, T-C, XI 2007.

Mycena zephirus (Fr. Fr.) P. Kumm - in litter, T-C, F-U, IX-X 2007.

Paxillus involutus (Batsch.: Fr.) Fr. s.l. - on the ground, T-C, X 2007.

Peniophora quercina (Pers.: Fr.) Cooke - fallen branches of Quercus robur, T-C, X 2007.

Phallus impudicus L.: Pers. - on the ground, T-C VII-VIII 2007.

Phlebia radiata Fr. - on lying trunks of Alnus glutinosa, T-C, VIII-XI 2007.

Phlebia tremellosa (Schrad.: Fr.) Nakasone \& Burds. - on stumps of deciduous tree, XI 2007.

Phleogena faginea (Fr.: Fr.) Link - on dead of deciduous tree, T-C, XII 2007.

Note. Species recorded in Poland in several tens localities (Szczepkowski, Chachuła 2010; Kujawa et al. 2012).

Pholiota lenta (Pers.: Fr.) Singer - on the ground, T-C, IX-XI 2007.

Pholiota mutabilis (Scop.: Fr.) P. Kumm. - on stumps of Alnus glutinosa, T-C, F-U, X 2007.

Pholiota squarossa (Weigel.: Fr.) P. Kumm. - on deciduoud wood, T-C, X 2007.

Piptoporus betulinus (Bull.: Fr.) P. Karst. - on birch wood, T-C, VI-XI 2007.

Pluteus atricapillus (Batsch) Fayod - on dead wood, T-C, F-U, X 2007.

Polyporus brumalis (Pers.) Fr. - on fallen branches, T-C, F-U, X-XII 2007.

Psathyrella vernalis Velen. - on the ground, among litter, T-C, IV-V 2007.

Pseudoclitocybe cyathiformis (Bull.: Fr.) Singer - on the ground, T-C, IX-XII 2007.

Psilocybe aeruginosa (M.A. Curtis: Fr.) Noordel. - on decayed wood, T-C, X 2007.

Psilocybe fascicularis (Huds.: Fr.) Noordel. - on stumps, T-C, F-U, VII-X 2007.

Psilocybe lateritia (Schaeff.: Fr.) Noordel. - on stumps of deciduous tree, T-C, F-U, IX-XI 2007.

Ramaria eumorpha (P. Karst.) Corner - on the ground, T-C, X 2007.

Ramaria stricta (Pers.: Fr.) Quél. - on litter, T-C, IX 2007.

Rhodocollybia butyracea for. asema (Fr.Fr.) Antonin, Halling\&\&Noordel. - on litter, T-C, F-U, X 2007.

Rickenella fibula (Bull.: Fr.) Raith. - among mosses, T-C, VI-VIII 2007.

Ripartites tricholoma (Alb. \& Schwein.: Fr.) P. Karst. - on the ground, F-U, IX-XII 2007.

Russula amoenolens Romagn. - on the ground, T-C, VIII-IX 2007.

Russula atrorubens Quél. - on the ground, T-C, IX 2007.

Russula emetica (Schaeff.) Pers.: Fr. - on the ground, T-C, F-U, X 2007.

Russula claroflava Grove - on the ground, T-C, X 2007.

Russula cyanoxantha (Schaeff.) Fr. - on the ground, T-C, X 2007.

Russula cyanoxantha var. peltereaui Singer - on the ground, T-C, X 2007.

Russula fragilis var. fragilis (Pers.: Fr.) - on the ground, T-C, X 2007.

Russula ochroleuca (Pers.) - on the ground, T-C, VII-XI 2007.

Russula olivascens (Pers.) Bres. - on the ground, T-C, X 2007.

Russula paludosa Britzelm - on the ground, T-C, X 2007.

Russula pectinatoides Peck - on the ground, T-C, VI 2007.

Russula solaris Ferd. \& Winge - on the ground, T-C, XI 2007.

Russula vesca Fr. - on the ground, T-C, VII-X 2007.

Russula viscida Kudřna - on the ground, T-C, X 2007.

Note. (Wojewoda 2003; Bujakiewicz 2004; Szkodzik 2005; Miśkiewicz 2000a; Gierczyk et al. 2009;

Karasiński 2009a).

Russula xerampelina (Schaeff.) Fr. - on the ground, T-C, VIII 2007.

Schizophyllum commune Fr.: Fr. - on stumps of deciduous tree, T-C, XII 2007.

Scleroderma bovista Fr. - on the ground, T-C, VIII 2007.

Scleroderma citrinum Pers. - on the ground, T-C, VII-IX 2007.

Scleroderma verrucosum (Bull.): Pers. - on the ground, T-C, VII 2007. 
Sparassis crispa (Wulf.): Pers. - on the ground, T-C, IX 2007.

Stereum hirsutum (Willd.: Fr.) Gray - on stumps of deciduous tree, T-C, X-XII 2007.

Stereum sanguinolentum (Alb. \& Schwein.: Fr.) Fr. - on branches of coniferous trees, T-C, XI 2007.

Strobilurus tenacellus (Pers.: Fr.) Singer - on fallen cones of Pinus sylvestris, V 2007.

Suillus luteus (L.: Fr.) Roussel - on the ground, under Pinus sylvestris, T-C, X 2007.

Trametes gibbosa (Pers.: Fr.) Fr. - on stumps of deciduous tree, T-C, X-XI 2007.

Trametes versicolor (L.: Fr.) Pilát - on stumps of deciduous tree, T-C, X-XI 2007.

Tricholoma sulphureum (Bull.: Fr.) P. Kumm. - on the ground, under Quercus robur, T-C X 2007.

Tubaria furfuracea (Pers. Fr.) Gillet - on fallen twigs, T-C, V 2007.

Tylopilus felleus (Bull.: Fr.) P. Karst. - on the ground, under Pinus sylvestris, T-C, VII 2007.

Typhula setipes (Grev.) Berthier - on fallen Alnus glutinosa leaves, F-U, F-A, XI 2007.

Xerocomus badius (Fr.: Fr.) Kühner ex. Gilbert - on the ground, T-C, IX-X 2007.

Xerocomus pascuus (Pers.) Krombh. - on the ground, T-C, X 2007.

Xerocomus subtomentosus var. subtomentosus (L.: Fr.) Quél. - on the ground, T-C, F-U, VII-IX 2007, IX 2008. Xeromphalina cornui (Quél.) J. Favre - on dead wood, T-C, IX 2007.

\section{CONCLUSIONS}

The undertaken studies in Białaczów forest reserve have shown that it is an area of high mycological diversity. Species composition of the reserve is influenced by factors like recreation usage of the area, the watercourse in it's northern part, and the community of Tilio-Carpinetum calamagrostietosum which is dominat in the reserve and riparian forests: Fraxino-Alnetum and Ficario-Ulmetum, growing in smaller amounts. The attempts to evaluate the diversity of macromycetes of the reserve, are interesting, more so because of the wind storm that had stricken the reserve in 2011, almost utterly destroying it. The received data can be the ground to evaluate the fungal changes of Białaczów reserve, and used for comparison with mycological studies in future.

Acknowledgements. We are grateful to the anonymous reviewer for helpful suggestions on the manuscript. The manuscript we dedicate to Professor Maria Lawrynowicz who nearly 50 years ago initiated research on macromycetes in the Central Poland.

\section{REFERENCES}

Adamczyk J. 2007. Grzyby wielkoowocnikowe rezerwatu Babsk w województwie łódzkim na tle przemian szaty roślinnej. Parki nar. i Rez. Przyr. 26 (2): 3-16

Breitenbach J., Kränzlin F.1984. Pilze der Schweiz I (Ascomyceten). Mykologia, Luzern.

Breitenbach J., Kränzlin F. 1986. Pilze der Schweiz II (Heterobasidiomycetes, Aphyllophorales, Gastromycetes). Mykologia, Luzern.

Breitenbach J., Kränzlin F.1991. Pilze der Schweiz III (Strobilomycetaceae und Boletaceae, Paxillaceae, Gomphidiaceae, Hygrophoraceae, Tricholomataceae, Polyporaceae). Mykologia, Luzern.

Breitenbach J., Kränzlin F.1995. Pilze der Schweiz IV (Entolonataceae, Pluteaceae, Amanitaceae, Agaricaceae, Coprinaceae, Bolbitiaceae, Strophariaceae). Mykologia, Luzern.

Breitenbach J., Kränzlin F. 2000. Pilze der Schweiz (Cortinariaceae). Mykologia, Luzern.

Bujakiewicz A. 2004. Grzyby wielkoowocnikowe Babiogórskiego Parku Narodowego. (In:) B. Wołoszyn, A. Jaworski, J. Szwagrzyk (eds). Babiogórski Park Narodowy. Monografia przyrodnicza. Komitet Ochrony Przyrody PAN, Kraków.

Bujakiewicz A. 2010. On some agarics occurring in carr forests. Acta Mycol. 45(1): 73-89.

Domański S. 1965. Flora Polska. Grzyby (Fungi) 2: Podstawczaki (Basidiomycetes), Bezblaszkowe (Aphyllophorales), Żagwiowate I (Polyporaceae I), Szczeciniakowate I (Mucronoporaceae I). PWN, Warszawa. 
Flisińska Z. 2004. Fungi in the Lublin Region. Macromycetes (Basidiomycetes) 2. Lubelskie Towarzystwo Naukowe, Lublin, 530 pp.

Galli R. 1996. Le Russule. Atlante praticomonografico per la determinazione. Edinatura, Milan.

Gierczyk B., Chachuła P., Karasiński D., Kujawa A., Pachlewski T., Snowarski M., Ślusarczyk T., Wójtowski M. 2009. Macrofungi of the Polish Bieszczady Mountains. Part. I. Parki nar. i Rez. Przyr. 28 (3): 3-100.

Index Fungorum, retrieved 10.12.2012 at www.indexfungorum.org

Jülich W. 1984. Die Nichtbläterpilze, Gallertpilze und Bauchpilze (Aphyllophorales, Heterobasidiomycetes, Gastromycetes). Kleine Kryptogamenflora II b/1. VEB Gustav Fischer Verlag Jena.

Karasiński D. 2009. Macrofungi of the „Ochojec” Nature Reserve. (In:) J.B. Parusel (ed.). Rezerwat przyrody „Ochojec” w Katowicach (Górny Śląsk). Monografia naukowo-dydaktyczna. Centrum Dziedzictwa Przyrody Górnego Śląska, Katowice: 86-103.

Kondracki J. 2002. Geografia regionalna Polski. Wydawnictwo Naukowe PWN, Warszawa: 257-260.

Knudsen H., Vesterholt J. (eds). 2008. Funga Nordica. Agaricoid, boletoid and cyphelloid genera. Nordsvamp, Copenhagen, $557 \mathrm{pp}$.

Kujawa A. 2012. Grzyby makroskopijne Polski w literaturze mikologicznej. (In:) M. Snowarski (ed.). Atlas grzybów Polski. Retrieved 15.12.2012, from the database at http://www.grzyby.pl/grzyby-makroskopijne-Polski-w-literaturze-mikologicznej.htm [in Polish].

Ławrynowicz M. 1973. Grzyby wyższe makroskopowe w grądach Polski Środkowej. Acta Mycol. 9 (2): 146-199.

Ławrynowicz M. 1989. Chorology of the European Hypogeous Ascomycetes. I. Elaphomycetales. Acta Mycol. 25 (1): 3-41.

Lawrynowicz M. 1990. Chorology of the European Hypogeous Ascomycetes. II. Tuberales. Acta Mycol. 26 (1): 7-75.

Ławrynowicz M. 2002. Grzyby. (In:) J.K. Kurowski (ed.). Parki Krajobrazowe Polski Środkowej. Przewodnik sesji terenowych, Uniwersytet Łódzki, Łódź: 36-38.

Miśkiewicz A. 2000. Rare, threatened and new for Poland macromycetes found in Bukowiec reserve (W Carpathians). Acta Mycol. 35 (2): 197-216.

Moser M. 1978. Die Röhrlinge und Blätterpilze (Polyporales, Boletaes, Agaricales, Russulales). Kleine Kryptogamenflora II b/2. VEB Gustav Fischer Verlag Jena.

Nespiak A. 1981. Flora Polska. Grzyby (Mycota) 14: Podstawczaki (Basidiomycetes), Bedłkowe (Agaricales), Zasłonakowatwe (Cortinariaceae), Zasłonak II (Cortinarius II). PWN, Warszawa - Kraków.

Nespiak A. 1990. Flora Polska. Grzyby (Mycota) 19: Podstawczaki (Basidiomycetes), Bedłkowe (Agaricales), Zasłonakowate (Cortinariaceae), Strzępiak (Inocybe). PWN, Warszawa - Kraków.

Noordeloos M.E., Kuyper T.W., Vellinga E.C. (eds). 2001. Flora Agaricina Neerlandica. Critical monographs on families of agarics and boleti occurring in the Netherlands. 5 Agaricaceae. A.A. Balkema Publishers, Rotterdam, 169 pp.

Noordeloos M.E., Kuyper T.W., Vellinga E.C. (eds). 2005. Flora Agaricina Neerlandica. Critical monographs on families of agarics and boleti occurring in the Netherlands. 6. Taylor \& Francis Group, $226 \mathrm{pp}$.

Operat Urządzeniowy lasu Leśnictwa Białaczów 1998.

Rąkowski G. (ed.). 2006. Rezerwaty przyrody w Polsce Środkowej. Instytut Ochrony Środowiska, Warszawa, 168 pp.

Skirgiełło A. 1960. Flora Polska. Grzyby (Mycota) 1: Podstawczaki (Basidiomycetes), Borowikowe (Boletales) PWN, Warszawa.

Skirgiełło A. 1991. Flora Polski. Grzyby (Mycota) 10: Podstawczaki (Basidiomycetes), Gołąbkowe (Russulales), Gołąbkowate (Russulaceae), Gołąbek (Russula). PWN, Warszawa - Kraków.

Skirgiełło A. 1998. Flora Polski. Grzyby (Mycota) 15: Podstawczaki (Basidiomycetes), Gołąbkowe (Russulales), Gołąbkowate (Russulaceae) II, Mleczaj (Lactarius). PAN, Instytut Botaniki im. W. Szafera, Kraków.

Szczepkowski A., Chachuła P. 2010. Nowe stanowiska i nowe gatunki żywicieli suchogłówki korowej Phleogena faginea (Fr.) Link w Polsce. Parki Nar. i Rez. Przyr. 29 (1): 93-121.

Szkodzik J. 2005. Macromycetes in communities of Abies alba on its range border in Central Poland. Acta Mycol. 40 (1): 113-131.

Wojewoda W. 2003. Checklist of Polish larger Basidiomycetes. (In:) Z. Mirek (ed.). Biodiversity of Poland 7. W. Szafer Institute of Botany, Polish Academy of Sciences, Kraków, 812 pp. 
Wojewoda W., Ławrynowicz M. 2006. Red list of the macrofungi in Poland. (In:) Z. Mirek, K. Zarzycki, W. Wojewoda, Z. Szeląg (eds). Red list of plants and fungi in Poland. W. Szafer Institute of Botany, Polish Academy of Sciences, Kraków: 53-70.

\section{Różnorodność gatunkowa makromycetes w rezerwacie Białaczów (Polska Środkowa)}

\section{Streszczenie}

Prezentowane dane są wynikiem badań prowadzonych w latach 2006-2007 w rezerwacie Białaczów (Polska Środkowa). Celem badań było poznanie składu gatunkowego grzybów makroskopowych i przedstawienie ich na tle drzewostanu i siedliska. Łącznie zidentyfikowano 167 taksonów grzybów makroskopowych (13 gatunków Ascomycota i 154 Basidiomycota). W rezerwacie, dominują grzyby saprotroficzne - 118 gatunków, grzybów mikoryzowych zebrano 44 gatunki, a pasożytniczych 5 gatunków. Pod względem ekologicznym przeważają grzyby tworzące owocniki na ziemi (74) oraz na drewnie (62), natomiast grzyby naściółkowe reprezentowane są przez 31 gatunków.

W rezerwacie odnotowano także jedenaście gatunków z czerwonej listy grzybów wielkoowocnikowych zagrożonych w Polsce: Phleogena faginea (E), Hypsizygus ulmarius, Mycena purpureofusca, Inonotus tomentosus (V), Clavariadelphus fistulosus, C. junceus, Gyroporus cyanescens, Lactarius chrysorrheus, Pachyphloeus melanoxanthus (R), Russula amoenoleus, Hygrophorus hypothejus var. hypothejus (I).

Przedstawione wyniki z jednej strony stanowią dokumentację mykologiczną, a z drugiej materiał wyjściowy do badań porównawczych w procesie zmian w następstwie zniszczenia rezerwatu przez wichurę w 2011 roku. 